\title{
Integral Sliding Mode Control of Airship Pitch Channel simplified model
}

\author{
Aijun Zhou ${ }^{1, a}$, Junbo $\mathrm{Chu}^{2}{ }^{\text {,Haibo Wang }}{ }^{1}$ \\ Tianwei $\mathrm{Li}^{1}$ and Guoqiang Liang ${ }^{3}$ \\ ${ }^{1}$ Department of navigation, Dalian Naval Academy \\ Dalian, 116018, China \\ ${ }^{2}$ Department of missile, Dalian Naval Academy \\ Dalian, 116018, China \\ ${ }^{3}$ Department of control engineering, Naval aeronautical and astronautical University \\ Yantai, 264001, China \\ azajwxy@163.com
}

Keywords: hypersonic aircraft; stability; control; free flying; numerical simulation

\begin{abstract}
A kind of integral type sliding mode control law was designed for a kind of simplified pitch channel model of hypersonic aircraft which is widely discussed recently. The advantage of sliding mode control is that it has strong robustness. And the integral sliding mode control method can reduce steady state error. And with the help of constructing two Lyapunov functions, all signals of close system are proved to be stable. At last, detailed simulation was done to testify the rightness of the proposed method.
\end{abstract}

\section{Introduction}

The control problem of hypersonic vehicle has attracted the interest of many researchers in the world. It is mainly due to the strong nonlinearity ${ }^{[1-4]}$, fast time-varying characteristics and the three channel coupling problems brings great challenge to the control theory. At present, the traditional methods such as traditional PID, state feedback and state space method ${ }^{[5-8]}$ have been adopted by scholars at home and abroad, also variable structure, adaptive control and other modern control methods are tried in hypersonic vehicle control, even neural network, genetic algorithm, fuzzy control and other intelligent method are studied and applied. At present, the complexity of the control of hypersonic vehicle leads to the robustness of the design of the general control method can not be guaranteed, which means that the stability margin is not enough. In this paper, an integral sliding mode control method is proposed for a class of hypersonic vehicle pitching channel model, which can not only ensure the robustness, but also reduce the steady state error due to the introduction of integral.

\section{Model Description}

A kind of hypersonic vehicle can be simplified as below differential equations to described its pitch channel movement:

$$
\begin{gathered}
\dot{V}=\frac{T \cos \alpha-D}{m}-g \sin \gamma \\
\dot{\gamma}=\frac{L+T \sin \alpha}{m V}-\frac{g \cos \gamma}{V}
\end{gathered}
$$




$$
\begin{aligned}
& \dot{\alpha}=q-\dot{\gamma} \\
& \dot{q}=\frac{M}{I} \\
& \dot{h}=V \sin \gamma \\
& \ddot{\eta}=-2 \varsigma \omega_{n} \dot{\eta}-\omega_{n}^{2} \eta+\omega_{n}^{2} \eta_{c}, \omega_{n}=5, \varsigma=0.7
\end{aligned}
$$

Where aero coefficient is identified as

$$
\begin{gathered}
L=\bar{q} S C_{L}, C_{L}=C_{L}^{\alpha} \alpha+C_{L}^{\delta} \delta+C_{L}^{0}, \quad T=\bar{q} S C_{T} \\
D=\bar{q} S C_{D}, C_{D}=C_{d}^{\alpha^{2}} \alpha^{2}+C_{D}^{\alpha} \alpha+C_{D}^{\delta^{2}} \delta^{2}+C_{D}^{\delta} \delta+C_{D}^{0} \\
M=\bar{q} S \bar{c}\left[C_{M \alpha}+C_{M \delta}+C_{M q}\right], C_{M \alpha}=C_{M \alpha}^{\alpha^{2}} \alpha^{2}+C_{M \alpha}^{\alpha} \alpha+C_{M \alpha}^{0}, \\
C_{M \delta}=C_{e}\left(\delta_{e}-\alpha\right), \\
C_{M \alpha}=10^{-4}\left(0.06-e^{-M_{a} / 3}\right)\left(-2 \alpha^{2}+120 \alpha-1\right) \\
C_{M q}=\frac{\bar{c} q}{2 V}\left(-0.025 M_{a}+1.37\right)\left(-0.0021 \alpha^{2}+0.0053 \alpha-0.23\right) \\
C_{M \delta}=0.0292(\delta-\alpha) \\
C_{L}=\alpha\left(0.493+1.91 / M_{a}\right) \\
C_{D}=0.0082\left(171 \alpha^{2}+1.15 \alpha+2\right)\left(0.0012 M_{a}^{2}-0.054 M_{a}+1\right) \\
C_{T}=\left\{\begin{array}{l}
38\left[1-164\left(\alpha-\alpha_{0}\right)^{2}\right]\left(1+17 / M_{a}\right)(1+0.15) \eta, \eta<1 \\
38\left[1-164\left(\alpha-\alpha_{0}\right)^{2}\right]\left(1+17 / M_{a}\right)(1+0.15 \eta), \eta<1
\end{array}\right\}
\end{gathered}
$$

And $V$ is speed, $\gamma$ is the speed angle, $\alpha$ is attack angle, $Q$ is the attitude angle speed, $h$ is the height. $\phi$ is the oil supplying factor, $\delta_{c}$ is the duck wing and $\delta_{e}$ is the lift rudder.

\section{Sliding mode controller design}

Define error variable as $e=\alpha-\alpha^{d}$, and choose a common integral sliding mode variable as

$$
s=e+c_{1} \int e d t
$$

Solve the derivative of sliding mode as

$$
\dot{s}=\dot{e}+c_{1} e
$$

Then it can be transformed as

$$
\dot{s}=\dot{\alpha}+c_{1} e=q-\dot{\gamma}+c_{1} e
$$

By using the information of above model, it can be written as

$$
\dot{s}=q-\frac{L+T \sin \alpha}{m V}+\frac{g \cos \gamma}{V}+c_{1} e
$$

And it can be rewritten as

$$
\dot{s}=q-\frac{T \sin \alpha}{m V}+\frac{g \cos \gamma}{V}+c_{1} e-\frac{\bar{q} S C_{L}}{m V}
$$

Finally, we get

$$
\dot{s}=q-\frac{T \sin \alpha}{m V}+\frac{g \cos \gamma}{V}+c_{1} e-\frac{\bar{q} S\left(C_{L}^{\alpha} \alpha+C_{L}^{\delta} \delta+C_{L}^{0}\right)}{m V}
$$


And define new variables as follows

$$
\begin{gathered}
l_{2}(\alpha, q, e)=q-\frac{T \sin \alpha}{m V}+\frac{g \cos \gamma}{V}+c_{1} e-\frac{\bar{q} S\left(C_{L}^{\alpha} \alpha+C_{L}^{0}\right)}{m V} \\
l_{1}=-\frac{\bar{q} S C_{L}^{\delta}}{m V}
\end{gathered}
$$

Then the derivative of sliding mode can be written as

$$
\dot{s}=l_{1} \delta+l_{2}(\alpha, q, e)
$$

Then the sliding mode control law can be designed as

$$
\delta=-\operatorname{sign}\left(l_{1}\right)\left[k_{1} s+k_{2} \frac{s}{|s|+k_{3}}+k_{4} s^{1 / 3}+k_{5} \int s d t\right](16)
$$

Where $k_{i}>0$, and assume there exist a big enough positive constant such $k_{6}$ and $k_{7}$ that

$$
\left|l_{2}(\alpha, q, e)\right|<k_{6}|s|+k_{7}(17)
$$

Choose a Lyapunov function as

$$
V=\frac{1}{2} s^{2}+\frac{k_{5}\left|l_{1}\right|}{2}\left(\int s d t\right)^{2}
$$

Then solve its derivative, it satisfies

$$
\begin{aligned}
& \dot{V}=s \dot{s}+k_{5}\left|l_{1}\right|\left(s \int s d t\right) \\
& =l_{1} \delta s+s l_{2}(\alpha, q, e)+k_{5}\left|l_{1}\right|\left(s \int s d t\right)
\end{aligned}
$$

And it can be written as

$$
\dot{V}=-\left|l_{1}\right|\left[k_{1} s^{2}+k_{2} \frac{s^{2}}{|s|+k_{3}}+k_{4} s^{4 / 3}+k_{5} s \int s d t\right]+s l_{2}(\alpha, q, e)+k_{5}\left|l_{1}\right|\left(s \int s d t\right)
$$

With the help of inequality method, it can be rewritten as

$$
\begin{aligned}
& \dot{V} \leq-\left|l_{1}\right|\left[k_{1} s^{2}+k_{2} \frac{s^{2}}{|s|+k_{3}}+k_{4} s^{4 / 3}+k_{5} s \int s d t\right]+k_{6} s^{2}+k_{7}|s|+k_{5}\left|l_{1}\right|\left(s \int s d t\right) \\
& =-\left|l_{1}\right|\left[k_{1} s^{2}+k_{2} \frac{s^{2}}{|s|+k_{3}}+k_{4} s^{4 / 3}\right]+k_{6} s^{2}+k_{7}|s|
\end{aligned}
$$

So it is easy to choose big enough parameters $k_{1}, k_{2}$ and $k_{3}$ such that

$$
\dot{V} \leq 0
$$

Then according to Lyapunov theory, we can prove $s \rightarrow 0$.

Since $c_{1}>0$, then it has

$$
e=-c_{1} \int e d t
$$

Choose another Lyapunov function as

$$
V_{a}=\left(\int e d t\right)^{2} / 2
$$

Then it holds

$$
\dot{V}_{a} \leq 0
$$

Then according to Lyapunov theory, we get $\int e d t \rightarrow 0$, so we have $e \rightarrow 0$, then finally we can 
prove that the system is stable.

\section{Numerical Simulation and result analysis}

Set model parameters as

$$
\begin{gathered}
I_{x x}=-7.1 * 10^{-5} \mathrm{~m}^{2}+19.1 \mathrm{~m}-59430 \\
I_{y y}=-8.03 * 10^{-4} \mathrm{~m}^{2}+219.74 m-1690000 \\
I_{z z}=-8.03 * 10^{-4} \mathrm{~m}^{2}+219.74 m-1690000 \\
I_{y y 0}=1.23 * 10^{7}, v_{s}=3.017 * 10^{2}, \rho_{a}=1.84 * 10^{2}, \\
g_{a}=9.7147, \quad h=30000 \\
V=4525, \eta_{c}=0.15662
\end{gathered}
$$

With above model and air coefficients and set initial condition as above paragraph and write a program with $m$ language in Matlab software, then the simulation can be done with above control law where desired value of attack angle is set as 4 degree, and simulation results can be shown as following figures.

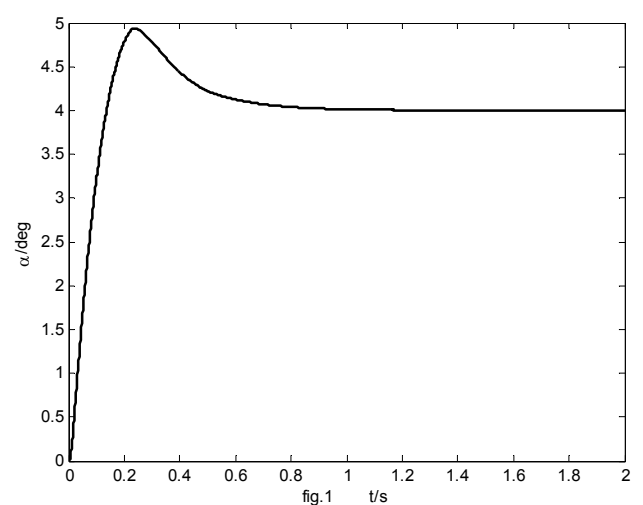

Fig 1 The curve of attack angle

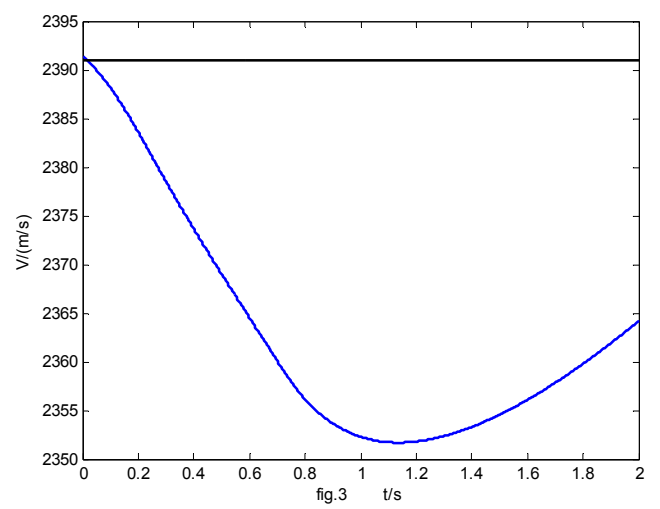

Fig 3 The curve of speed

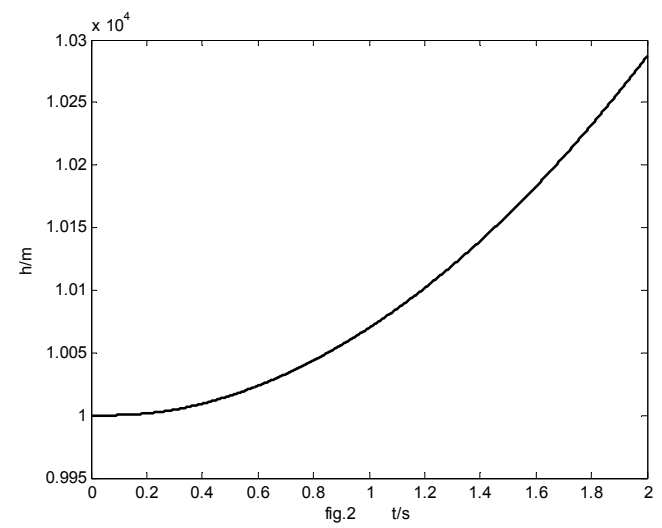

Fig 2 The curve of the height

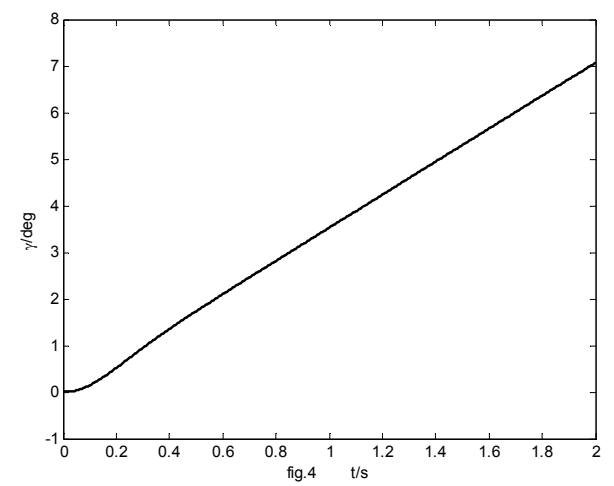

Fig 4 The curve of speed angle 


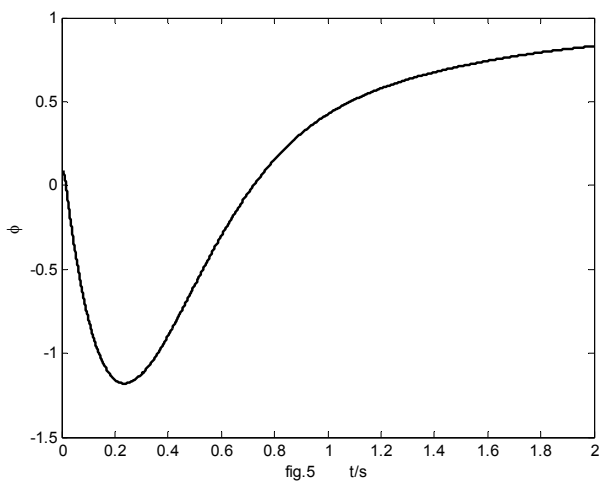

Fig 5 The curve of oil supplying factor

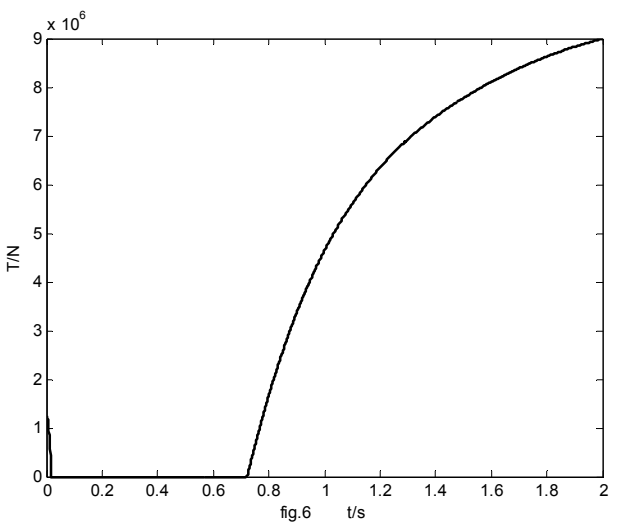

Fig 6 The curve of thrust

We can make a conclusion that the proposed method has good swiftness according to above simulation result, and the response time of attack angle is about $0.3 \mathrm{~s}$. And the height and speed of aircraft are in a normal range. So above simulation result shows the proposed sliding mode control method is effective for this kind of aircraft.

\section{Conclusion}

A kind of integral sliding mode control method is proposed to solve the attack angle tracking problem of a kind of simplified model of pitch channel of hypersonic aircraft systems. All signal of close system are guaranteed to be bounded and stable by constructing Lyapunov function method. At last, detailed numerical simulation result shows that the proposed method is right and effective, which also has good swiftness.

\section{References}

[1]Buschek $\mathrm{H}$ and Calise A J.Hypersonic flight control system design using fixed order robust controllers[A].In:AIAA Guidance,Navigation and Control Conference[C].Chattanooga,TN, AIAA 95-6062

[2]Buschek H, Calise A J. Uncertainty modeling and fixed-order controller design for a hypersonicvehicle model. Journal of Guidance, Control and Dynamics, 1997, 20(1): 42-48.

[3]Heller $\mathrm{M}$ and Sachs G.Flight dynamics and robust control of a hypersonic test vehicle with ramjet propulsion[A].In:AIAA International Space Planes and Hypersonic Systems and Technologies Conference[C].Norfolk,VA,AIAA 98-1521

[4]M. Ohno, Y. Yamaguchi, T. Hata, M. Takahama, Robust flight control law design for an automatic landing flight experiment [J], Control Engineering Practice, 1999, 7(9):1143-1152

[5]Naidu S D,Banda S S and Buffington J L.Unified approach to $\mathrm{H}_{2}$ and $\mathrm{H}_{\infty}$ optimal control of a hypersonic vehicle[A].In:Proceedings of the American control conference[C].San Diego, California,1999,vol.4:2737-2741.

[6]Christopher I. Marrison. synthesis of robust control systems for a hypersonic aircraft decision and contro1. IEEE, 1994。4:3324-3329

[7]E. mooij. linear quadratic regulator reentry control-performance assessment using a taguchi approach. AIAA, international space planes and hypersonic systems and technologies conference. 1998: 665-677.

[8]Kevin. P. Groves, Andrea. Serranti, Stephen. Yurkovich. Anti-Windup Control for an Air-breathing Hypersonic Vehicle Model. AIAA, 2006-6557: 1-14. 